\title{
Contact Pressure Between the Vocal Folds in Reinke's Edema: Experimental Observations on an Excised Human Larynx
}

\author{
${ }^{*}$ Fabrice Silva, ${ }^{\dagger}$ Thierry Legou, ${ }^{\ddagger}$ Pierre Champsaur, ${ }^{\dagger, \S}$ Antoine Giovanni, and ${ }^{\ddagger, \pi}$ Aude Lagier, ${ }^{*} \ddagger \S$ Marseille, and \\ $\dagger$ Aix-en-Provence, France, and $\llbracket$ Liège, Belgique
}

\begin{abstract}
Summary: Objective. To analyze the range of values of the contact pressure between the membranous vocal folds with Reinke's edema and to compare it to those observed in the absence of such a lesion.

Methods. Two human larynges were separately tested on the experimental bench, one of them with a bilateral loose swelling of the vocal folds. Once in a glottal prephonatory configuration, airflow was increased until achievement of self-sustained oscillations while recording aerodynamic, acoustic, electroglottographic data, and contact pressure between the folds.

Results. We observed well-documented variations in acoustical parameters, as the decrease of the fundamental frequency and the increase of the phonation threshold pressure. The results of the study also point to a significant increase in the amplitude of the contact pressure in presence of the Reinke's edema, and a lower degree of harmonicity of the produced sounds.

Conclusion. This is the first report of ex vivo study of a larynx with Reinke's edema. It highlights the increase in the contact pressure during phonation, which possibly contributes to sustain the lesion once it appeared.
\end{abstract}

Key Words: Excised human larynges-Contact stress-Reinke's edema-Phonotrauma.

\section{INTRODUCTION}

Reinke's edema is a common exudative lesion of the Reinke's space, ${ }^{1}$ presenting as a unilateral or bilateral, sessile, swelling of the vocal fold filled with fluid, and very mobile during phonation. ${ }^{2}$ The main risk factors for Reinke's edema are active smoking and vocal abuse ${ }^{3,4}$ whereas the main factor of other exudative lesions of Reinke's space (nodules and polyps) is the vocal abuse/misuse. According to Verdolini et $\mathrm{al}^{5}{ }^{5}$ the link between vocal abuse/misuse and lesions would be the phonotrauma defined as the mechanical stress applied to the vocal folds during phonation with a biological response of the tissues. Titze enumerates various components that could be involved in phonotrauma: tensile stress, active contractile stress in muscle, inertial stress, arytenoid contact stress, shear stress at anterior and posterior macula flava, aerodynamic stress, and collision stress between vocal folds. ${ }^{6}$ Among these, the collision stress has been studied using several approaches: measurements in ex vivo experiments using full mammals larynges ${ }^{7,8}$ or hemilarynges, ${ }^{9}$ and estimation based on in vivo observations on human subjects. ${ }^{8,10-13}$ In particular, the consequence of an organic lesion such as Reinke's edema on the collision stress is still unreported. The orientation of the elastin fibers in the Reinke's space lead Dikkers and Nikkels to the hypothesis that the forces applied to the vocal fold act

\footnotetext{
Accepted for publication February 20, 2020.

From the *Aix Marseille Universite, CNRS, Centrale Marseille, LMA, Marseille, France; $†$ Aix Marseille Universite, CNRS, LPL, Aix-en-Provence, France; $\ddagger$ Aix Marseille Universite, APHM, Laboratoire d'Anatomie, Faculté de Médecine, Marseille, France; §AP-HM, CHU Conception, Service d'ORL, Marseille, France; and the ๆService d'ORL, CHU de Liège, Liège, Belgique.

Address correspondence and reprint request to Fabrice Silva, Laboratory of Mechanics and Acoustics, 4 impasse Nikola Tesla, CS40006, 13453 Marseille cedex 13, France. E-mails: silva@lma.cnrs-mrs.fr, thierry.legou@lpl-aix.fr,

pierre.champsaur@mail.ap-hm.fr, Antoine.GIOVANNI@ap-hm.fr,

aude.lagier@chuliege.be

Journal of Voice, Vol. $\mathbf{\square}$, No. $\mathbf{\square}$, pp. $\mathbf{\square}-\mathbf{\square}$ 0892-1997

(C) 2020 The Voice Foundation. Published by Elsevier Inc. All rights reserved. https://doi.org/10.1016/j.jvoice.2020.02.020
}

more likely as tearing forces that are initially directed medially and, during phonation, cranially and craniolaterally. ${ }^{3}$

In vivo experiments on a patient with a polyp ${ }^{10}$ exhibited impact stress in the mid-third of the membranous vocal folds with an initial peak of $25 \mathrm{kPa}$ at the onset and a maximum of $4 \mathrm{kPa}$ during steady phonation, whereas it ranged from less than $1 \mathrm{kPa}$ to almost $4 \mathrm{kPa}$ for subjects without benign mass lesion.

This paper proposes to describe the impact stress at midthird of the membranous vocal folds in a pathological excised larynx with Reinke's edema and to compare it to a normal excised larynx in similar conditions.

\section{MATERIAL AND METHODS}

\section{Larynges}

Two female human larynges were harvested from fresh cadavers, within 24 to 48 hours after death, according to French law. One was normal, and the other was pathological with a bilateral loose swelling of the vocal folds (Figure 1) diagnosed as bilateral grade 1 Reinke's edema ${ }^{14}$ At the time of death, the age of the persons was 76 and 72, respectively. Larynges were rapidly frozen in $0.9 \%$ saline solution, thawed overnight also in saline solution and cleaned with removal of the extraneous tissues and supraglottic structures (epiglottis and vestibular folds) before the experiment, according to the literature. ${ }^{15}$ The glottal prephonatory configuration was then achieved using a stitch around the vocal processes of the arytenoids in order to create arytenoid adduction, and bilateral thyroplasty was performed in order to fully adduct the membranous part of the vocal folds using Montgomery implants. ${ }^{16}$ No crico-thyroid tilt was applied.

\section{Experimental set-up}

The setup is based on the one detailed in. ${ }^{17}$ The larynx is placed horizontally, the posterior edges of the thyroid 

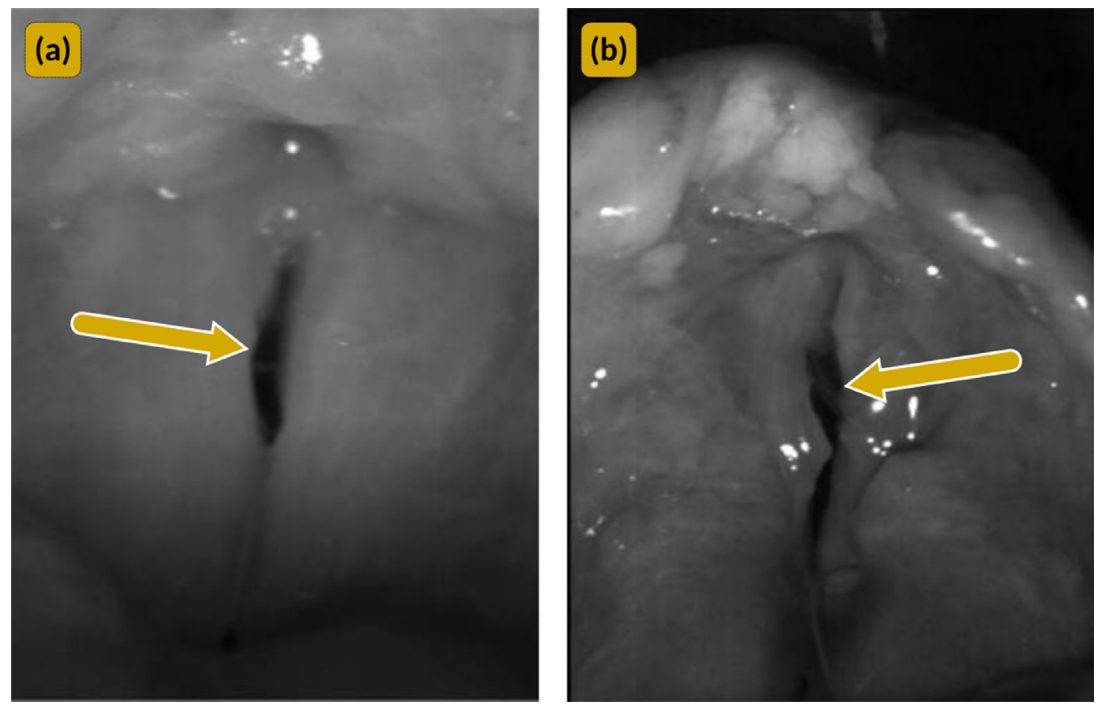

FIGURE 1. Top views of the normal larynx (a) and of the larynx with Reinke's edema (b). Arrows point to the location of the contact force transducer. Images recorded just before the beginning of the experiment.

laminae being laid on the support, and the cricoid can move freely, without any mechanical constraint. Air supply is provided by a turbine (Werei Rietschle, Fürstenfeldbruck, Germany), and connected through a flexible tube ( $3 \mathrm{~m}$ long, diameter $40 \mathrm{~mm}$ ) to a humidifier (Aquador, Dräger) and then to the upper trachea through an intubation tube (Rüsch Elit tracheal tube cuffed, inner diameter $6.5 \mathrm{~mm}$ ), Figure 2. The intubation tube is sealed to the trachea by inflating the cuff. The airflow is controlled in a kind of Mesa di Voce singing exercise (progressively increased, maintained, then decreased), scanning the subglottal pressure range for human phonation. Precautions are taken to slowly go across onset and offset thresholds, in order to avoid the bifurcation delay effect. ${ }^{18}$

\section{Signal processing}

The following signals are simultaneously recorded with a $6250 \mathrm{~Hz}$ sampling frequency using the Phonedit software (Signaix, Aix en Provence, France) driving the EVA workstation (SQLab, Aix-en-Provence, France).
Subglottal pressure (denoted $\mathrm{P}_{\mathrm{SG}}$ hereafter) is accessed inserting through the crico-thyroid membrane a venous catheter (23-gauge) connected at the other end to the calibrated pressure sensor of the EVA workstation. The signal is then filtered (analog low-pass filter with a $47 \mathrm{~Hz}$ cutoff frequency) in order to only retain the aerodynamic part of the $\mathrm{P}_{\mathrm{SG}}$ and not the acoustic component.

The ElectroGlottographic signal (EGG) is collected using electrodes placed on the anterior aspect of the thyroid laminae and connected to the Laryngograph microProcessor (Wallington, United Kingdom). RMS values of the EGGs are computed accounting for $20 \mathrm{~ms}$ frames with $75 \%$ overlap (done using Praat, ${ }^{19}$ same windowing parameters used for all RMS computations), along with the time variation of the fundamental frequency $\mathrm{f}_{\mathrm{o}}$ (using the YIN algorithm with a tolerance on confidence set to $\left.0.5^{20}\right)$.

The radiated acoustic pressure $\left(\mathrm{P}_{\mathrm{ext}}\right)$ is captured using a microphone (AKG C520) placed $30 \mathrm{~cm}$ away from the larynx, slightly higher than the larynx to avoid turbulent air noise, and is used to compute the sound pressure level $\mathrm{L}_{\mathrm{p}}$.

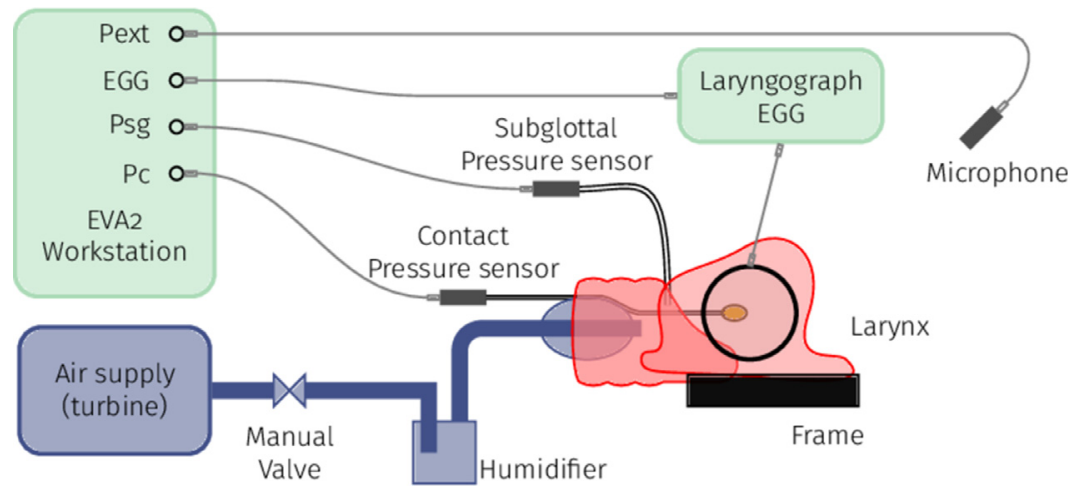

FIGURE 2. Schematic of the excised larynx experimental set-up. Larynx is highlighted in red.

EGG, electroglottograph; PSG, subglottal pressure. (For interpretation of the references to color in this figure legend, the reader is referred to the Web version of this article.) 
Finally, a piezo-resistive transducer (Model 105, Precision Measurement Company, Ann Arbor; linear range 0 -30 PSI $=0-207 \mathrm{kPa} ; 2.67 \mathrm{~mm}$ diameter and $0.508 \mathrm{~mm}$ thickness) is placed between the vocal folds to estimate the contact pressure. A high-pass analog filter (1st order analog with $1.5 \mathrm{~Hz}$ cutoff frequency) is required for signal conditioning and removes the mean value. Nevertheless the absolute contact pressure can be retrieved by assuming that the contact pressure vanishes during the opened glottis episode. RMS values of the absolute contact pressure (denoted $\mathrm{P}_{\mathrm{c}}$ ) can thus be estimated and provide insight about the effective stress applying on the tissues.

Several criteria were considered for the estimation of the onset and offset phonation thresholds. The most consistent appeared to be based on the threshold value of $65 \mathrm{~dB}$ SPL for the sound pressure level. The high level of aerodynamic noise before phonation explains the relatively high value of this threshold. The magnitude of the contact pressure for these estimated thresholds is thus non-negligible and is also reported in the Results section.

An additional indicator for harmonicity (denoted Harm.) is obtained by using the comb filtering method similar to the one described in. ${ }^{21}$ It indicates the importance of the energy contained in periodic component relatively to the total energy of the frame (ie, a value of 1 means that the signal is perfectly periodic with very low noise, while 0 may relate to a very low signal to noise ratio but also to a strong quasiperiodic component). The same rationale is used to determine the proportion of the energy into subharmonics (SubHarm.), the remaining contribution being considered as broadband noise.

\section{RESULTS}

Figure 3 shows the processed descriptors for the healthy and the pathological larynges, in the left and right columns respectively and the numerical values are presented in Table 1, mentioning mean values and standard deviations computed from the observations on the various runs. The phonation onset threshold of the healthy larynx is characterized by a subglottal pressure of $2.78 \pm 0.35 \mathrm{hPa}$, a fundamental frequency of $253.8 \pm 2.0 \mathrm{~Hz}$, and an amplitude of contact pressure of $18.5 \pm 1.0 \mathrm{kPa}$. Above the onset threshold, the progressive increase of the subglottal pressure (up
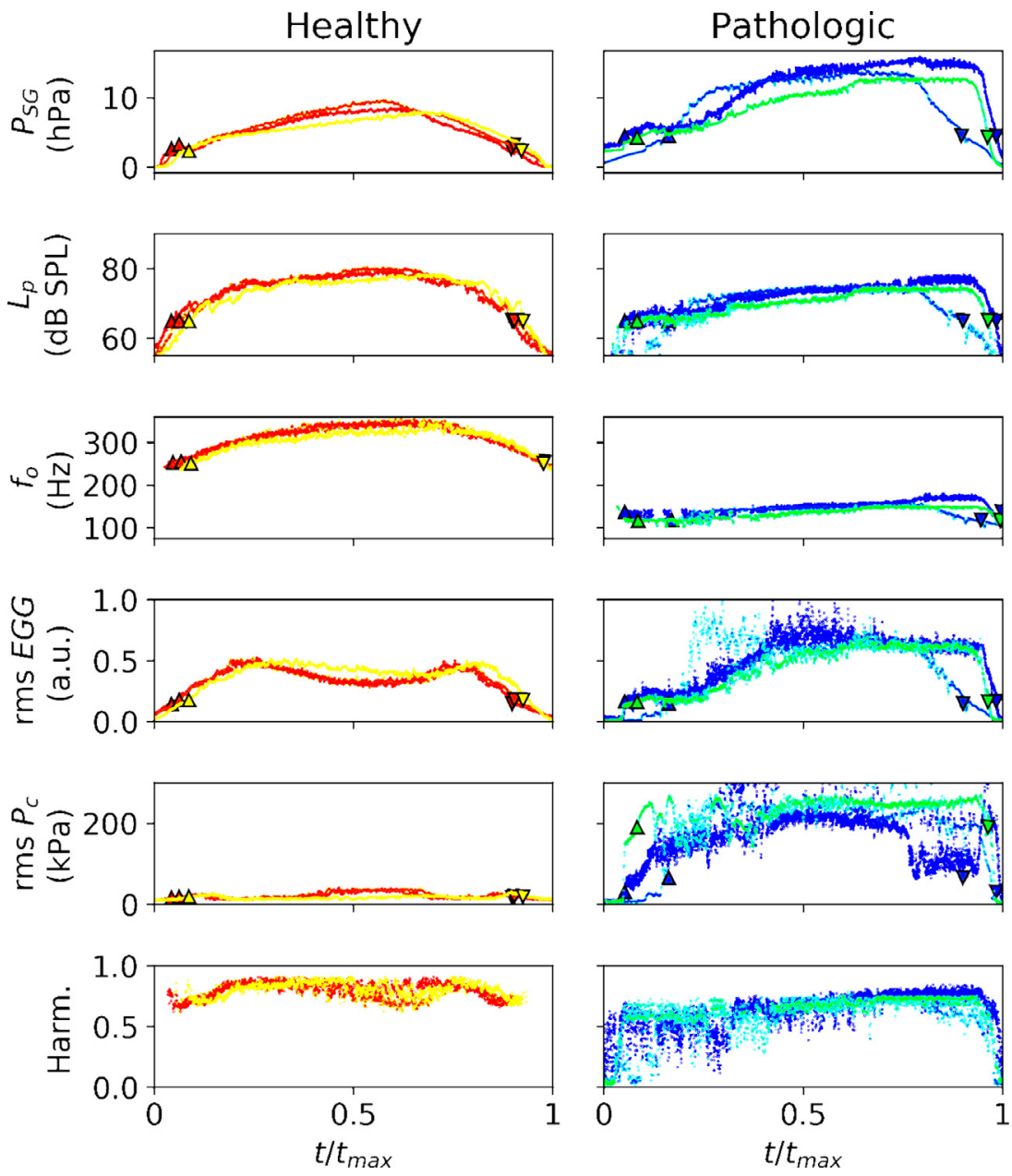

FIGURE 3. Examples of time variations of the subglottal pressure (PSG), sound level (Lp), fundamental frequency (fo) and RMS values of the electroglottograph (EGG) and contact pressure (Pc) and harmonicity (from top to bottom), for three tokens for the healthy larynx (in the left column) and for the pathological one (in the right column). 
TABLE 1.

Values of Subglottal Pressure ( $\left.P_{s G}\right)$, Fundamental Frequency $\left(f_{o}\right)$ and Sound Level $\left(L_{p}\right)$, of the RMS Values of Electroglottograph (EGG) and Contact Pressure $\left(P_{c}\right)$ at Onset and Offset Threshold and Maximum Operating Point

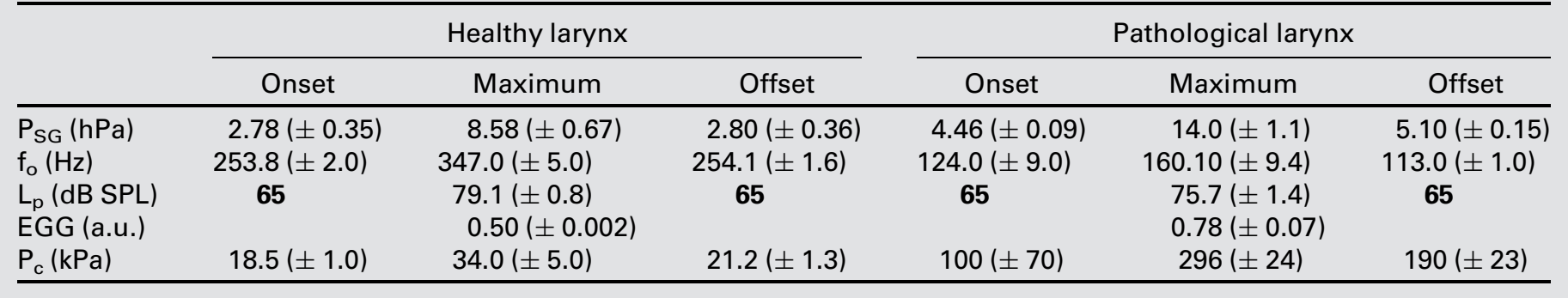

to $8.58 \pm 0.67 \mathrm{hPa}$ ) leads to monotonic variations of the fundamental frequency (up to $347.0 \pm 5.0 \mathrm{~Hz}$ ) and sound level ( $\mathrm{L}_{\mathrm{p}}$ up to $\left.79.1 \pm 0.8 \mathrm{~dB} \mathrm{SPL}\right)$. On the contrary, the evolution of the EGG is not monotonous, with a maximum amplitude (below 0.50 a.u.) obtained before the maximal subglottal pressure. The maximal contact pressure observed is $34.0 \pm 5.0 \mathrm{kPa}$. The phonation remains when decreasing the subglottal pressure until reaching the offset threshold (also defined by the $65 \mathrm{~dB}$ SPL level) characterized by a subglottal pressure of $2.80 \pm 0.36 \mathrm{hPa}$, a fundamental frequency of $254.1 \pm 1.6 \mathrm{~Hz}$, and an amplitude of contact pressure of $21.2 \pm 1.3 \mathrm{kPa}$.

Concerning the pathological larynx, the phonation onset is estimated for a subglottal pressure of $4.46 \pm 0.09 \mathrm{hPa}$, a fundamental frequency of $124.0 \pm 9.0 \mathrm{~Hz}$ and an amplitude of the contact pressure of $100 \pm 70 \mathrm{kPa}$. The subglottal pressure reaches then higher values (up to $14.00 \pm 1.10 \mathrm{hPa}$ ), with maximal values for fundamental frequency $160 \pm$ $10 \mathrm{~Hz}$, intensity $75.7 \pm 1.4 \mathrm{~dB}$ SPL. After some transient where the vibration is unstable, RMS values of EGG and
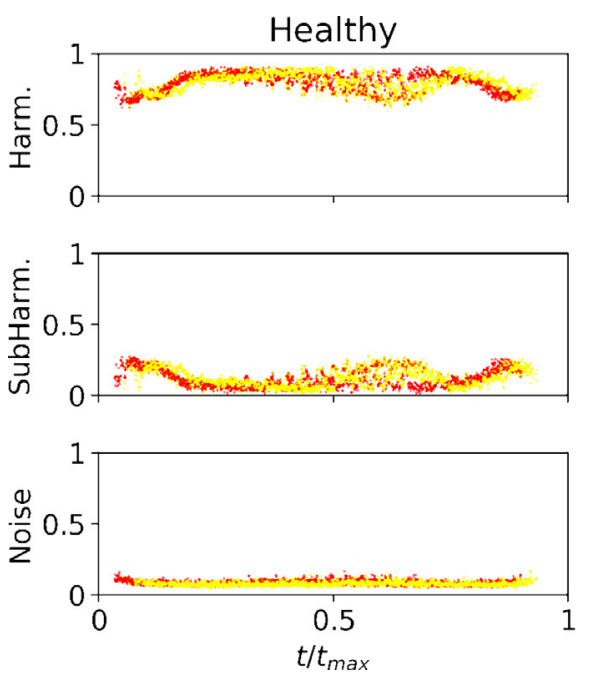

contact pressure reach plateaux at $0.78 \pm 0.07$ a.u. and 296 $\pm 24 \mathrm{kPa}$, respectively. The phonation offset is attained for a subglottal pressure of $5.10 \pm 0.15 \mathrm{hPa}$, with a fundamental frequency of $113.0 \pm 1.0 \mathrm{~Hz}$ and an amplitude of contact pressure of $190 \pm 23 \mathrm{kPa}$.

The last row of Figure 3 reports the level of harmonicity of the produced (radiated) sounds. It is further detailed in Figure 4 which highlights the distribution of energy between the periodic oscillation (fundamental and harmonics), the subharmonics (which accounts for instability of the vibration, eg, period doubling) and noise. In the healthy larynx, most of the energy is contained in the harmonics (between $60 \%$ and $90 \%)$, a little part in subharmonics $(<25 \%$, essentially in transient phases) and almost no noise $(<10 \%)$. On the contrary, in the pathological larynx, energy is not as high in harmonics $(40 \%-80 \%)$, and a significant level of energy is supported by subharmonics $(<15 \%)$ and noise (up to $40 \%)$. Note that the variance of these estimators depends on the jitter of the signal, ie, the dispersion of the points in Figure 4 is probably due to a higher jitter in the pathologic larynx.

FIGURE 4. Repartition of energy into harmonic structure (top), subharmonics (middle) and noise (bottom) for healthy larynx (left) and pathological one (right). Variance of these estimators (showing as "noise" on the curves) depends on the stationarity of the signal, and may increase, for example, with jitter and shimmer. 


\section{DISCUSSION}

The acoustical parameters are consistent with classical knowledge on patients suffering from Reinke's edema (see, eg, ref. $^{22}$ ), especially concerning the lowering of the fundamental frequency that is involved in the common voice-induced gender confusion of patients with Reinke's edema. ${ }^{23}$

The harmonicity of the healthy larynx was higher than the pathologic one (Figure 3). The loss of harmonicity of the healthy larynx phonation was essentially due to the transient emergence of subharmonics and noise remained very low. On the contrary, the pathologic larynx presented lower harmonicity, with both higher subharmonics and higher noise.

Even if typical values of contact pressure are not established in healthy subjects, the main observation is the extremely high level of contact pressure during stable phonation (up to $296 \mathrm{kPa}$ ) in the pathological larynx, compared to the healthy larynx (no more than $34 \mathrm{kPa}$ ).

A previous in vivo study highlighted the differences between the quasistatic contact pressure (induced by the prephonatory configuration) in healthy subjects and one pathological subject suffering from a polyp. ${ }^{10}$ The subject with a polyp presented a transient increase in contact pressure at the onset of phonation, followed by a slightly higher contact pressure during sustained phonation in comparison to the healthy condition. This laryngeal behavior is very different from the observations reported in the pathological larynx of the current study which produced very high levels of the dynamical contact pressure (related to vibrations of the vocal folds) all along the phonation trials. We can hypothesize that this homogeneity in the contact pressure is correlated to the shape of the lesion of Reinke's edema which is very homogenous and widespread contrary to a polyp which is localized and mobile leading to more unstable contact between the vocal folds. The Reinke's edema increases the mass of the vocal fold, leading to increased inertia and collision force (ie, dynamical contact pressure).

The design of the study is also very important: in vivo experiments measure the effects of the anatomical perturbations (passive component), and of the compensation mechanisms (active components), whereas ex vivo studies allow the analysis of the isolated effect of the passive, geometric component. Hence, the present ex vivo design suggests that the increase in the contact pressure is here essentially due to the changes in the physical properties of the vocal folds induced by the pathology.

In order to minimize the difference induced by the experimental setup, we chose not to apply any tension of the vocal folds, ie no crico-thyroid tilt which may not be the same in the two larynges. We chose to perform a direct adduction of the membranous part of the vocal folds with the Montgomery implants because their effect was easier to control. This may explain the relative instability of vibration even in the healthy larynx: we reproduced the effects of a paralysis of the external laryngeal nerve. To our best knowledge, all previous studies in excised human or animal larynges introduced a degree of vocal fold tension in order to improve the adduction of their membranous part (for review, see ref. ${ }^{24}$ ).
A limit of the present study is that only the contact pressure was measured, although it is only a partial approach of the phonotrauma. Further studies may in particular focus on the shearing stress which may also be an important component of the physiopathology of exudative lesions of Reinke's space.

\section{CONCLUSION}

This study is the first ex vivo study of a larynx with Reinke's edema. It highlighted the increase in the contact pressure during phonation. Although the role of phonotrauma is not as prominent as in other exudative lesions of the Reinke's space, it suggests that the phonotrauma may maintain the lesion after its appearance.

\section{SUPPLEMENTARY DATA}

Supplementary data related to this article can be found online at https://doi.org/10.1016/j.jvoice.2020.02.020.

\section{REFERENCES}

1. Remacle M, Degols JC, Delos M. Exudative lesions of Reinke's space. An anatomopathological correlation. Acta Otorhinolaryngol Belg. 1996;50:253-264

2. Dikkers FG, Nikkels PG. Benign lesions of the vocal folds: histopathology and phonotrauma. Ann Otol Rhinol Laryngol. 1995;104(9 Pt 1):698-703

3. Dikkers FG, Nikkels PG. Lamina propria of the mucosa of benign lesions of the vocal folds. Laryngoscope. 1999;109:1684-1689.

4. Marcotullio D, Magliulo G, Pezone T. Reinke's edema and risk factors: clinical and histopathologic aspects. Am J Otolaryngol. 2002;23:81-84.

5. Verdolini K, Rosen CA, Branski RC, et al. Shifts in biochemical markers associated with wound healing in laryngeal secretions following phonotrauma: a preliminary study. Ann Otol Rhinol Laryngol. 2003;112:1021-1025

6. Titze IR. Mechanical stress in phonation. J Voice. 1994;8:99-105.

7. Verdolini K, Chan R, Titze IR, et al. Correspondence of electroglottographic closed quotient to vocal fold impact stress in excised canine larynges. J Voice. 1998;12:415-423.

8. Jiang JJ, Shah AG, Hess MM, et al. Vocal fold impact stress analysis. $J$ Voice. 2001;15:4-14.

9. Jiang JJ, Titze IR. Measurement of vocal fold intraglottal pressure and impact stress. J Voice. 1994;8:132-144.

10. Hess MM, Verdolini K, Bierhals W, et al. Endolaryngeal contact pressures. J Voice. 1998;12:50-67.

11. Gunter HE, Howe RD, Zeitels SM, et al. Measurement of vocal fold collision forces during phonation: methods and preliminary data. $J$ Speech Lang Hear Res. 2005;48:567-576.

12. Chen Li-J, Mongeau L. Verification of two minimally invasive methods for the estimation of the contact pressure in human vocal folds during Phonation. J Acoust Soc Am. 2011;130:1618-1627.

13. Verdolini K, Hess MM, Titze IR, et al. Investigation of vocal fold impact stress in human subjects. JVoice. 1999;13:184-202.

14. Tan M, Bryson PC, Pitts C, et al. Clinical grading of Reinke's edema. Laryngoscope. 2017;127:2310-2313.

15. Alipour F, Finnegan EM, Jaiswal S. Phonatory characteristics of the excised human larynx in comparison to other species. $J$ Voice. 2013;27:441-447.

16. Lagier A, Guenoun D, Legou T, et al. Control of the glottal configuration in ex vivo human models: quantitative anatomy for clinical and experimental practices. Surg Radiol Anat. 2017;39:257-262.

17. Legou T, Lagier A, Silva F, et al. Test bench for human excised larynx studies. In: Manfredi C, ed. Models and Analysis of Vocal Emissions 
for Biomedical Applications: 9th International Workshop. Firenze: Firenze University Press; 2015.

18. Bergeot B, Almeida A, Gazengel B, et al. Response of an artificially blown clarinet to different blowing pressure profiles. J Acoust Soc Am. 2014;135:479-490.

19. Boersma P, Weenink D. Praat: Doing Phonetics by Computer. (version 6.1.03); 2019. http://www.praat.org/.

20. de Cheveigné A, Kawahara H. YIN, a fundamental frequency estimator for speech and music. J Acoust Soc Am. 2002;111:1917-1930.

21. Kasuya H, Ogawa S, Kikuchi Y. An adaptive comb filtering method as applied to acoustic analyses of pathological voice. IEEE International
Conference on Acoustics, Speech, and Signal Processing (ICASSP '86). IEEE; 1986:669-672.

22. Hirano M, Tanaka S, Fujita M, et al. Fundamental frequency and sound pressure level of phonation in pathological states. $J$ Voice. 1991;5(2):120-127.

23. Matar N, Portes C, Lancia L, et al. Voice quality and gender stereotypes: a study of lebanese women with Reinke's edema. J Speech Lang Hear Res. 2016;59(6):S1608-S1617.

24. Garcia M, Herbst CT. Excised larynx experimentation: history, current developments, and prospects for bioacoustic research. Anthropol Sci. 2018;126(1):9-17. 